\title{
A Role Casting Method Based on Emotions in a Story Generation System
}

\author{
Ruck Thawonmas $^{1}$, Masanao Kamozaki ${ }^{1}$, and Yousuke Ohno ${ }^{1}$ \\ Intelligent Computer Entertainment Laboratory \\ Graduate School of Science and Engineering, Ritsumeikan University \\ Kusatsu, Shiga, 525-8577, Japan \\ ruck@ci.ritsumei.ac.jp
}

\begin{abstract}
We first point out a problem in the role casting method of a story generation system called OPIATE and then propose a solution to this problem. The existing casting method does not take into account the emotions of a cast non-player character towards actions that it must perform in its role, leading to a miscast. In the proposed casting method, such emotions are considered, besides emotions towards other characters as done in the existing one. Evaluation results, using an online-game simulator, confirm the effectiveness of the proposed method over the existing one with respect to the number of miscasts.
\end{abstract}

\section{Introduction}

Storytelling pervades our daily life and has a large social impact through media such as novels, movies, dramas, and games. It thus plays a central role in an entertainment area.

In our research, we focus on a story generation system called OPIATE[1] [2] oriented to online-games. In OPIATE, a story based on the Propp's morphology 3 is developed through interactions between a player character (PC) and nonplayer characters (NPCs). In an OPIATE's story, a quest is issued to the PC acting as the hero, and NPCs act according to their cast roles, such as the villain, princess, etc.

The main technology in OPIATE is case-based reasoning (CBR) 4 used for selection of a sub-plot (case) that defines a set of actions to be carried out by the corresponding characters, each cast a different role. At a given point in the story, a selected case is the one with the highest suitability of roles and actions.

In this paper, we point out a problem residing in role casting of OPIATE. We then propose a new casting method for solving the problem and evaluate its effectiveness.

\section{Story Generation}

According to the Propp's morphology, there are 31 functions, each for being carried out by a character cast one out of seven roles. These functions differ in 
their effects on the sub-plot. Their examples are "trickery" carried out by the villain attempting deception to prepare for the act of villainy and "complicity" by the hero succumbing to the trickery. In OPIATE, two roles were additionally added, i.e., the family and the king. These 31 functions were thus divided into eight role groups of NPCs, and one role group of the PC, which is the hero. Henceforth, the term "character(s)" is used to represent both the PC and NPCs when there is no need to distinguish them.

An OPIATE's case is a script consisting of multiple lines. Each line represents a function and contains a maximum of three elements: the function name, an action that is carried out by the character cast the role corresponding to this function, and a character that is the objective of the action (henceforth, this kind of character is called objective character). Hence, a function involves up to two roles, depending on the presence of the last element.

\subsection{Case Suitability}

During case selection using CBR, the suitability of a case is computed as the average of the suitability of all functions in the case as follows:

$$
S n=\sum_{i=1}^{L n}(W r * S r i+W a * S a i) / L n
$$

where $S n$ denotes the suitability of case $n, L n$ represents the number of functions in this case, $W r$ and $W a$ are the weights. Sri is the sum of the relevance degrees to the role(s) in function $i$ of all available characters, where the relevance degree to a role of a character is decided based on the casting rules in Table 1. Sai is the sum of the relevance degrees to the action in function $i$ of all available characters' actions, where the relevance degree of a character's action to a function's action is 1 if the latter action can be accomplished by the former action and is 0 otherwise.

\subsection{Conflict Casting}

In OPIATE, once a case with the highest suitability has been selected, all roles in that case are cast to characters according the casting rules in Table 1, where roles are filled from top to bottom. As can be seen from this table, casting is based mainly on NPCs' emotions towards other characters. Let us take Villain for example. The role of Villain consists of aggressive and/or villainous actions such as Attack, Capture, and Expel. Its casting rule is to cast Villain to an NPC who has a negative emotion towards the Hero. Suppose that there exists an NPC with the most negative emotion towards the Hero, but this NPC does not like to carry out such aggressive and/or villainous actions. According to the casting rule, Villain will be cast, however, to this NPC, which is conflicting to its personality.

As can been seen from the above example, existing casting rules sometimes cast roles to NPCs that confict their personalities. 
Table 1. Casting rules

\begin{tabular}{|c|l|}
\hline Role & Rule \\
\hline Hero & The player character \\
\hline Villain & An NPC with a negative emotion towards the Hero \\
\hline Donor & $\begin{array}{l}\text { An NPC who has not met the Hero yet or } \\
\text { an NPC with a slightly positive emotion towards the Hero }\end{array}$ \\
\hline Mediator & An NPC with a slightly negative emotion towards the Hero \\
\hline Family & An NPC with a positive emotion towards the Hero \\
\hline Princess & $\begin{array}{l}\text { An NPC with a positive emotion towards the Hero } \\
\text { and a negative emotion towards the Villain }\end{array}$ \\
\hline Helper & $\begin{array}{l}\text { An NPC who has met Hero earlier and } \\
\text { has a positive emotion towards the Hero }\end{array}$ \\
\hline King & An NPC with a positive emotion towards the Princess \\
\hline False Hero & An NPC with a negative emotion towards the Hero \\
\hline
\end{tabular}

\subsection{Proposed Casting Method}

Here, we propose a new casting method that considers also NPCs' emotions towards actions. In the proposed casting method, for each role, a number of candidate NPCs are selected from the remaining NPCs according to the casting rules in Table 1. Then from these candidates, the NPC with the most positive emotions towards the role's actions is selected for being cast. However, casting will not be done if there is no NPC with positive emotions towards the role's actions among the candidates.

For example, let us take the case of Villain again. First, all remaining NPCs with a negative emotion towards the Hero are raised as the candidates for this role. Then, among these candidates, the NPC with the most positive emotions towards Villain's actions is selected for being cast. Thereby, with the proposed casting method, role casting is done without any conflict to NPCs' personalities.

\section{Implementation of Emotions}

In this section, we describe EMAI (Emotionally Motivated Artificial Intelligence) [5] 6] that we adopt for implementing NPCs' emotions.

\subsection{Emotions in Appraisal Space}

EMAI is a mechanism that decides agents' actions based on their emotions. There are two groups of emotions. One is a group of emotions related to internal desires of the agent (NPC) such as hungry or sleepy. The other one is a group of emotion towards other characters, objects, actions, and situations. Below we explain the implementation of the emotions towards other characters and actions because they are used in our research. Emotions in EMAI are defined by six orthogonal appraisal dimensions identified in [7]: Pleasantness, Responsibility, Certainty, Attention, Effort, and Control. Figure 1 shows typical emotions in the appraisal space spanned by Pleasant and Control. 


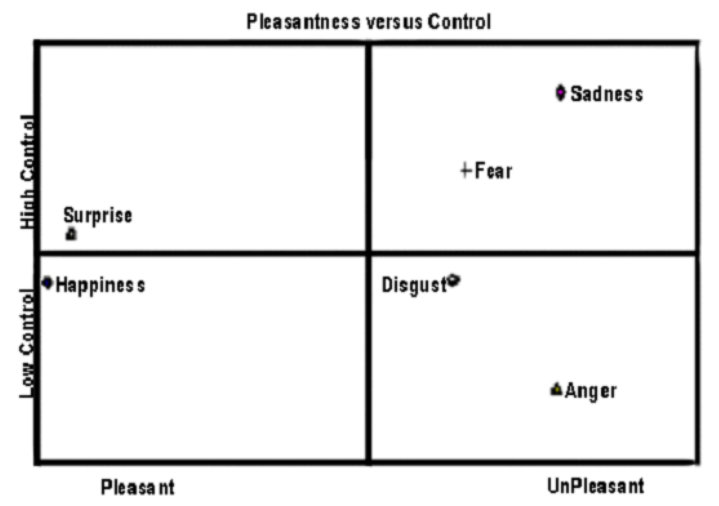

Fig. 1. Typical emotions in two dimensional appraisal space

\subsection{Emotional State and Its Updating Rule}

An event in the story occurs when a function in a selected case is carried out by the corresponding NPC, causing a change in the emotional state of the NPC towards each element of the event. In our system, an event $E$ is defined by at most two elements: an action and an objective character if this action acts upon someone. Let an element in $E$ be denoted by $e$. The NPC emotional state towards $e, \Omega_{e}$, is defined by the six orthogonal appraisal dimensions as follows:

$$
\Omega_{e}=\left\{P_{e}, R_{e}, C_{e}, A_{e}, E_{e}, O_{e}\right\},
$$

where the value on each dimension is represented by the initial character of the corresponding dimension, except for Control represented by $O$.

When an event occurs at time $t$, the NPC emotional state can be described by the following formula.

$$
\Omega_{E, t}=\sum_{e=1}^{n} W_{e} \Omega_{e, t},
$$

where $n$ is the number of elements in $E$ at time $t$ (either one or two), and $W_{e}$ is the weight of $e$. Let us denote the outcome of $E$ by $\Omega_{F}=\left\{P_{F}, R_{F}, C_{F}, A_{F}, E_{F}, O_{F}\right\}$ that is an emotion based on the action result and/or the feedback from the objective character. Because $\Omega_{F}$ indicates a right direction in the appraisal space that $\Omega_{E}$ should follow, the updating rule of each element in the NPC emotional state, henceforth called emotional element, can be given below as:

$$
\Omega_{e, t+1}=\Omega_{e, t}+W_{e}\left(\Omega_{F, t}-\Omega_{E, t}\right)
$$

Note that after the update, the direction of each emotional element will have been partly shifted to the direction of the event's outcome.

In Table 1 negative or positive emotions towards other characters are used for role casting. Such emotions are derived as follows. If the emotional element of an NPC towards a given character is nearer to Happiness than Sadness, then the 
NPC has a positive emotion towards that character, otherwise it has a negative emotion towards the character. The emotional element of an NPC towards a given action is decided in the same fashion

\section{Performance Evaluation}

In our performance evaluation, we focused on the Villain that has several functions and the Helper that has a high effect on the Hero. Using an online-game simulator, we compare the proposed casting method and the existing one with respect to the number of miscasts.

\subsection{Simulator}

In this simulator, we implemented one PC and 19 NPCs. The PC was also simulated and thus not directly controlled by a human player. NCPs' actions, their success/failure conditions, and the probability of receiving each feedback are summarized in Table 2. For Attack, the objective character is randomly selected from all other characters, while for Heal it is randomly selected from all characters, i.e., an NPC can carry out Heal to heal itself. For Quest and Give, the objective character is the PC. Capture and Save are not carried out by NPCs, but the emotional states towards them of the NPC issuing a quest Abduction or Potion Procurement in Table 3 to the PC are indirectly updated when the PC completes the quest.

Each NPC has three parameters: the number of items, the number of quest types, and its power. The number of item shows the number of items that an NPC of interest is currently holding, and this number is decremented each time it carries out Give. The number of quest types indicates how many quest types an NPC can issue to the PC among four types shown in Table 3. The power of an NPC is decreased accordingly when it is attacked by another NPC or a monster, and its power will be resumed to the initial value if it is successfully healed. For each NPC, the number of items and quest types were randomly initialized to a value between 0 and 4 and a value between 0 and 2, respectively. The initial power was randomly initialized to a value between 50 and 150 .

Among the four actions available, an NPC stochastically selects an action to carry out with the highest probability for the action towards which it has the most positive emotion and with the lowest probability for the action towards which it has the most negative emotion. For each NPC, each dimension of its emotional elements towards other characters and actions was initialized with a random value between -1.5 and 1.5. As described in 3.2, the emotional elements of an NPC are updated according the outcome of an event that is based on the action result (c.f. Table 4) and/or the feedback from the objective character (c.f. Table 5). NPCs who are attacked or healed also update their related emotional elements, i.e., for Attack the emotional elements of the objective NPC towards Attack and the character carrying out Attack are shifted to Sadness, and for Heal the emotional elements of the objective NPC towards Heal and the character carrying out Heal are shifted to Happiness provided that the heal is successful. 
Table 2. List of NPCs' actions, their success (failure) conditions, and the probability of each feedback

\begin{tabular}{|c|c|c|c|}
\hline Action & Role & Success(Failure) Condition & Feedback Probability \\
\hline Attack & Villain & $\begin{array}{c}\text { The power of the objective character becomes zero } \\
\text { (remains more than zero). }\end{array}$ & $\begin{array}{l}\mathrm{p}(\text { Sadness })=0.5 \text { and } \\
\mathrm{p}(\text { no feedback })=0.5\end{array}$ \\
\hline Heal & Helper & $\begin{array}{c}\text { The power of the objective character is recovered } \\
\text { to its initial value (does not change). }\end{array}$ & $\begin{array}{c}\mathrm{p}(\text { Happiness })=0.5 \text { and } \\
\mathrm{p}(\text { no feedback })=0.5 \\
\text { when the heal is successful only }\end{array}$ \\
\hline Quest & Donor & The PC accepts (refuses) to pursue the quest. & - \\
\hline Capture & Villain & Not considered. & - \\
\hline Save & Helper & Not considered. & - \\
\hline Give & Donor & $\begin{array}{l}\text { The NPC receives the Happiness (Sadness) } \\
\text { feedback from the PC. }\end{array}$ & - \\
\hline
\end{tabular}

Table 3. List of quests and their effects on the corresponding emotional elements

\begin{tabular}{|l|l|}
\hline Quest & Update direction when the PC completes (fails) the quest \\
\hline Expedition & $\begin{array}{l}\text { Shifted to Happiness (Sadness) the emotional elements } \\
\text { of the NPC towards Attack and the PC }\end{array}$ \\
\hline Abduction & $\begin{array}{l}\text { Shifted to Happiness (Sadness) the emotional elements } \\
\text { of the NPC towards Capture and the PC }\end{array}$ \\
\hline Potion Procurement & $\begin{array}{l}\text { Shifted to Happiness (Sadness) the emotional elements } \\
\text { of the NPC towards Heal and the PC }\end{array}$ \\
\hline Rescue & $\begin{array}{l}\text { Shifted to Happiness (Sadness) the emotional elements } \\
\text { of the NPC towards Save and the PC }\end{array}$ \\
\hline
\end{tabular}

For updating emotional elements, the weight $W_{e}$ was set to 0.5. The appraisal vectors of the two emotions in use were set according to [7, i.e., Happiness = $\{-1.46,0.09,-0.46,0.15,-0.33,-0.21\}$, and Sadness $=\{0.87,-0.36,0,-0.21,-0.14$, $1.15\}$.

When an NPC carries out Attack, if the objective character is a monster, the NPC continues fighting against the opponent monster, initialized with the power of 100, until the power of either of them reaches zero. The amount of damage that the NPC gives to or receives from the monster was set to a random value between 30 and 40. If the objective character is another NPC, the NPC carrying out Attack gives the damage to the objective NPC with a random value between 30 and 40.

The probability that Heal, Quest, and Give are successful was set to 0.5. The probability that the $\mathrm{PC}$ successfully completes a quest was also set to 0.5. Note that a quest will cause the issuing NPC to update its emotions at most twice, i.e., when the PC accepts or rejects to pursue the quest (c.f. Table 4), and then when the quest is completed or failed by the PC (c.f. Table 3). The latter update is an indirect one because the $\mathrm{PC}$ carries out an issued quest, but it is the issuing NPC that updates its emotional elements towards the PC and the related action. The weight for this indirect update $W_{e}$ was set to 0.25 . 
Table 4. List of actions' effects on the corresponding emotional elements

\begin{tabular}{|l|l|}
\hline Action & Update direction when successful (failed) \\
\hline Attack & $\begin{array}{l}\text { Shifted to Happiness (Sadness) the emotional element } \\
\text { of the NPC towards Attack }\end{array}$ \\
\hline Heal & $\begin{array}{l}\text { Shifted to Happiness (Sadness) the emotional element } \\
\text { of the NPC towards Heal }\end{array}$ \\
\hline Quest & $\begin{array}{l}\text { Shifted to Happiness (Sadness) the emotional elements } \\
\text { of the NPC towards Quest and the PC }\end{array}$ \\
\hline Give & $\begin{array}{l}\text { Shifted to Happiness (Sadness) the emotional elements } \\
\text { of the NPC towards Give and the PC }\end{array}$ \\
\hline
\end{tabular}

Table 5. List of feedbacks' effects on the corresponding emotional elements

\begin{tabular}{|l|l|}
\hline Action & Update direction when receiving a feedback \\
\hline Attack & $\begin{array}{l}\text { Shifted to Sadness the emotional element of the NPC } \\
\text { towards Attack and the objective character }\end{array}$ \\
\hline Heal & $\begin{array}{l}\text { Shifted to Happiness the emotional elements of the NPC } \\
\text { towards Heal and the objective character }\end{array}$ \\
\hline
\end{tabular}

\subsection{Results and Discussions}

In each trail the simulator was run for 150 seconds. After this, role casting for the eight NPC roles was done based on NPCs' emotional states according to the proposed method and the existing one. For comparisons, the number of trials was set to 200 , from which the number of miscasts of each method was obtained. Note that in the existing method, a miscast will occur if a cast NPC has negative emotions towards the actions of its role. In the proposed method, this type of miscasting does not occur, but there exist miscasts due to not able to cast a role to any candidate NPC if they all have negative emotions towards the role's actions.

Figures 2 and 3 show the time series of the distances to Happiness and Sadness from each emotional element of an NPC towards Player (the PC) and towards Attack and Villain, respectively, in a trail where the proposed method correctly cast Villain to this NPC. In our simulator, the emotional element towards Villain is the sum of the emotional elements towards the two related actions: Attack and Capture. Table 6 shows the action logs of this NPC.

The NPC received the Sadness feedback from the PC at $t=7$ and 45 . As a result, as shown in Fig. 2, the distance to Sadness of its emotional state towards Player decreased, while the distance to Happiness of this emotional state increased. This means that its emotion towards Player became more negative after these time steps.

In addition, the NPC successfully performed Attack at $t=6,30,33,34,42,83$, 105, 148, and 150. As shown in Figure 3 these successes resulted in having more positive emotions towards Attack and Villain. Note that at $t=6$ the distances to Happiness and Sadness of its emotional state towards Attack both decreased. However, because the decreasing amount of the former is larger than that of the 


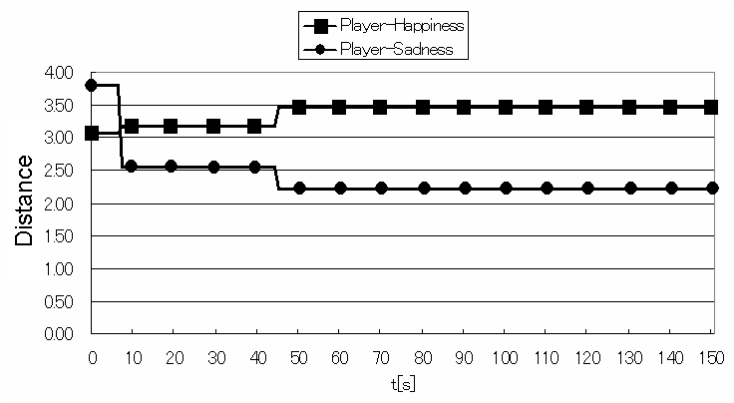

Fig. 2. Time series of the distances to Happiness and Sadness from the emotional element of an NPC towards Player

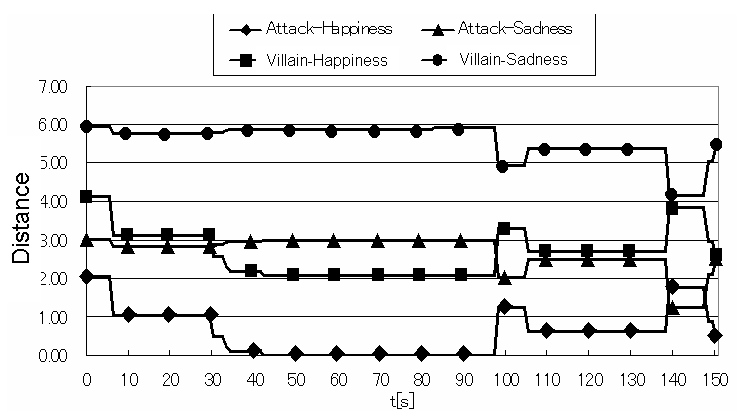

Fig. 3. Time series of the distances to Happiness and Sadness from the emotional elements of the NPC in Fig. 2 towards Attack and Villain

latter, this led to a more positive emotion towards Attack. At $t=98$ the NPC was attacked by NPC 15, and at $t=139$ its Attack was lost to a monster. As a result, after these time steps, its positive emotion towards Attack was decreased. It can be seen from these results that the NPC correctly updated its emotional states according to event outcomes.

Table 7 compares the existing casting method and the proposed casting method with respect to the number of miscasts. As can been seen from the table, the proposed method significantly outperforms the existing one. In both methods, the number of miscasts for Helper is more than that for Villain. This is due to the fact that Helper is cast after Villain because the casting rules in Table 1 are executed from top to bottom, which means that the number of NPCs available for Helper is less than that for Villain.

Table 8 shows results from a trial where the existing method miscast Villain to NPC 9, but the proposed method correctly cast Villain to NPC 6, where each number shows the distance to Happiness or Sadness of the emotional element of the corresponding NPC towards Player, Villain, or Helper. Because NPC 9 has the most negative emotion towards Player, Villain was cast to it in the existing method although it has a negative emotion towards the role. In the proposed 
Table 6. Action logs of the NPC in Figs. 2 and 3

\begin{tabular}{|c|c|}
\hline Action & $@$ Time (s) \\
\hline Successfully performed Attack to a monster & 6 \\
\hline Performed Give to the PC but received Sadness & 7 \\
\hline Successfully performed Attack to a monster & 30 \\
\hline Successfully performed Attack to a monster & 33 \\
\hline Successfully performed Attack to a monster & 34 \\
\hline Successfully healed by NPC 19 & 38 \\
\hline Successfully performed Attack to a monster & 42 \\
\hline Performed Give to the PC but received Sadness & 45 \\
\hline Successfully performed Attack to a monster & 83 \\
\hline Successfully healed by NPC 11 & 87 \\
\hline Attacked by NPC 15 & 98 \\
\hline Successfully performed Attack to a monster & 105 \\
\hline Successfully healed by NPC 12 & 108 \\
\hline Performed Attack to a monster but was lost to it & 139 \\
\hline Successfully healed by itself & 146 \\
\hline Successfully performed Attack to a monster & 148 \\
\hline Successfully performed Attack to a monster & 150 \\
\hline
\end{tabular}

Table 7. Comparison between the existing casting method and the proposed casting method with respect to the number of miscasts

\begin{tabular}{|l|c|c|}
\hline Role & The existing method & The proposed method \\
\hline Villain & 25 & 2 \\
\hline Helper & 28 & 4 \\
\hline
\end{tabular}

Table 8. Results from a trial where the existing method miscast Villain to NPC 9, but the proposed method correctly cast Villain to NPC 6

\begin{tabular}{|c|c|c|c|c|c|c|}
\cline { 2 - 7 } \multicolumn{1}{c|}{} & \multicolumn{2}{c|}{ Player } & \multicolumn{2}{c|}{ Villain } & \multicolumn{2}{c|}{ Helper } \\
\cline { 2 - 7 } \multicolumn{1}{c|}{} & Happiness & Sadness & Happiness & Sadness & Happiness & Sadness \\
\hline NPC1 & 2.26 & 3.73 & 3.04 & 4.06 & 3.45 & 4.37 \\
\hline NPC2 & 1.81 & 2.53 & 3.63 & 2.39 & 2.07 & 4.36 \\
\hline NPC3 & 1.44 & 2.69 & 1.90 & 4.55 & 1.97 & 4.21 \\
\hline NPC4 & 3.27 & 1.55 & 4.27 & 2.84 & 2.26 & 3.61 \\
\hline NPC5 & 1.89 & 2.24 & 3.64 & 2.07 & 2.50 & 3.99 \\
\hline NPC6 & $\mathbf{3 . 4 6}$ & 2.21 & 2.13 & $\mathbf{3 . 8 8}$ & 3.22 & 4.61 \\
\hline NPC7 & 2.29 & 1.46 & 2.77 & 2.61 & 3.51 & 4.26 \\
\hline NPC8 & 1.57 & 1.98 & 3.04 & 4.70 & 3.12 & 5.33 \\
\hline NPC9 & $\mathbf{3 . 6 1}$ & $\mathbf{1 . 0 4}$ & $\mathbf{4 . 0 7}$ & 2.84 & 2.87 & 4.95 \\
\hline NPC10 & 1.81 & 1.71 & 2.26 & 3.88 & 3.30 & 3.63 \\
\hline NPC11 & 2.03 & 1.62 & 2.57 & 3.53 & 3.16 & 4.48 \\
\hline NPC12 & 3.07 & 1.65 & 4.39 & 2.97 & 2.44 & 4.45 \\
\hline NPC13 & 2.27 & 1.67 & 3.27 & 4.14 & 3.65 & 4.43 \\
\hline NPC14 & 1.31 & 2.90 & 2.61 & 3.41 & 2.43 & 3.11 \\
\hline NPC15 & 1.49 & 2.95 & 2.70 & 3.19 & 3.18 & 4.11 \\
\hline NPC16 & 2.27 & 1.46 & 2.66 & 2.63 & 2.01 & 4.42 \\
\hline NPC17 & 2.98 & 2.75 & 4.51 & 2.90 & 3.85 & 5.25 \\
\hline NPC18 & 2.09 & 4.30 & 3.70 & 2.18 & 3.95 & 3.53 \\
\hline NPC19 & 2.08 & 2.41 & 2.87 & 3.32 & 1.94 & 5.18 \\
\hline
\end{tabular}


Table 9. Results from a trial where the proposed method miscast Helper

\begin{tabular}{|c|c|c|c|c|c|c|}
\cline { 2 - 7 } \multicolumn{1}{c|}{} & \multicolumn{2}{c|}{ Player } & \multicolumn{2}{c|}{ Villain } & \multicolumn{2}{c|}{ Helper } \\
\cline { 2 - 7 } \multicolumn{1}{c|}{} & Happiness & Sadness & Happiness & Sadness & Happiness & Sadness \\
\hline NPC1 & 1.61 & 2.45 & 3.13 & 4.04 & 2.16 & 4.57 \\
\hline NPC2 & 2.78 & 1.80 & 2.40 & 3.25 & 2.07 & 3.95 \\
\hline NPC3 & 1.73 & 2.28 & 1.86 & 4.77 & 1.76 & 4.05 \\
\hline NPC4 & 1.93 & 1.77 & 2.56 & 3.63 & 3.08 & 4.15 \\
\hline NPC5 & 2.32 & 1.41 & 4.60 & 2.33 & 2.66 & 4.46 \\
\hline NPC6 & 3.81 & 3.08 & 4.25 & 3.03 & 3.17 & 4.56 \\
\hline NPC7 & 2.15 & 2.30 & 2.66 & 2.57 & $\mathbf{4 . 1 0}$ & $\mathbf{3 . 9 6}$ \\
\hline NPC8 & 3.21 & 1.00 & 3.01 & 4.77 & 3.18 & 5.18 \\
\hline NPC9 & 4.11 & 1.39 & 2.93 & 3.02 & 2.86 & 5.01 \\
\hline NPC10 & 2.05 & 2.49 & 2.91 & 3.68 & $\mathbf{3 . 3 0}$ & $\mathbf{3 . 2 6}$ \\
\hline NPC11 & 1.81 & 2.32 & 3.49 & 2.82 & 1.75 & 4.05 \\
\hline NPC12 & 3.07 & 1.65 & 4.02 & 3.16 & 2.50 & 4.49 \\
\hline NPC13 & 2.27 & 1.67 & 3.50 & 3.45 & 3.65 & 5.36 \\
\hline NPC14 & 2.11 & 1.83 & 4.47 & 2.86 & 2.45 & 3.64 \\
\hline NPC15 & 3.33 & 1.65 & 4.07 & 2.49 & 2.37 & 4.40 \\
\hline NPC16 & 2.60 & 1.17 & 3.28 & 2.15 & 2.07 & 4.39 \\
\hline NPC17 & 3.30 & 1.56 & 4.56 & 2.91 & 1.84 & 4.36 \\
\hline NPC18 & 2.53 & 4.12 & 3.48 & 2.16 & 3.95 & 3.84 \\
\hline NPC19 & 3.61 & 3.13 & 2.67 & 3.98 & 2.68 & 4.17 \\
\hline
\end{tabular}

method, Villain was cast to NPC 6 that has a negative emotion towards Player and a positive emotion towards this role.

Table 9 shows results from a trial where the proposed method miscast Helper. The remaining candidates for Helper are NPC 7 and NPC 10 that both have a positive emotion towards Player. However, both have a negative emotion towards this role, so no casting was performed leading to a miscast. All other miscasts in the proposed method occurred in a similar fashion.

\section{Conclusions and Future Work}

To reduce miscasts in a story generation system OPIATE, we have proposed a role casting method that considers the emotions of NPCs towards both characters and roles. EMAI was adopted for implementing those emotions. The proposed method was evaluated with an online-game simulator. According to the evaluation results, the proposed method had a much lower number of miscasts than the existing method used in OPIATE, and miscasts in the proposed method were due to the unavailability of candidate NPCs with positive emotions towards the corresponding character and role's actions.

As our future work, we plan to implement the proposed method into an online game under development at our laboratory and evaluate it through real test plays. In addition, we plan to improve the formula for computing the suitability of a case and evaluate it on the aforementioned online game.

\section{References}

1. Fairclough, C.R., Cunningham, P.: A MULTIPLAYER OPIATE. International Journal of Intelligent Games \& Simulation 3(2), 54-61 (2004)

2. Fairclough, C.R.: Story Games and the OPIATE System Using Case-Based Planning for Structuring Plots with an Expert Story Director Agent and Enacting them in a Socially Simulated Game World. Doctoral Thesis, University of Dublin - Trinity College (2004) 
3. Propp, V.: Theory and History of Folklore. University of Minnesota Press (1984)

4. Aamodt, A., Plaza, E.: Case-Based Reasoning: Foundational Issues, Methodological Variations, and System Approaches. Artificial Intelligence Communications. 7(1), 39-52 (1994)

5. Baillie-de, B.P.: Programming Believable Characters for Computer Games. Charles River Media, pp. 385-392 (2004)

6. Baillie-de, B.P.: A Six Dimensional Paradigm for Generating Emotions in Virtual Characters. International Journal of Intelligent Games and Simulation. 2(2), 72-79 (2003)

7. Smith, C.A., Ellsworth, P.C.: Attitudes and Social Cognition. Journal of Personality and Social Psychology. 48(4), 813-838 (1985) 